\title{
Machine learning molecular dynamics studies of clay minerals
}

\author{
M. OKUMURA ${ }^{1 *}$, K. KOBAYASHI ${ }^{2}$, A. YAMAGUCHI ${ }^{1}$, H. \\ NAKAMURA $^{1}$, M. ITAKURA $^{1}$, M. MACHIDA $^{1}$ \\ ${ }^{1}$ Japan Atomic Energy Agency, Kashiwa, Chiba, JAPAN \\ ${ }^{2}$ Research Organization for Information Science and \\ Technology, Tokai, Ibaraki, JAPAN \\ * Corresponding author: okumura.masahiko@jaea.go.jp
}

Molecular dynamics (MD) simulations play an important role for investigating microscopic properties of clay minerals. There are two main methods of MD, i.e., classical MD and $a b$ initio MD. The computational cost of the former method is low, but the accuracy strongly depends on the empirical force fields used. On the other hand, the latter method does not have empirical parameters, so it is referred as "ab initio" or "first-principles". But the numerical cost of $a b$ initio MD is quite high. Therefore, it is difficult to perform large scale simulations using the ab initio MD.

Recently, a new MD method named "machine learning molecular dynamics" (MLMD) was proposed [1,2]. This method enables us to perform highly accurate MD simulations with low computational costs by exploiting machine learning techniques such as artificial neural networks (ANN) [1] or Gaussian Process (GP) regressions [2]. The principle of MLMD is simple. An ANN or GP is trained using data obtained by $a b$ initio calculations to reproduce a potential energy surface (PES), and low cost MD simulations are performed using the PES. Therefore, MLMD is highly accurate, comparable to $a b$ initio MD simulations, and without the high cost of $a b$ initio calculations.

However, MLMD is not omnipotent. One weakness is the explosion of the input dimension with respect to the number of atom types in the target system. This implies that MLMD is not suitable for MD simulations of systems with many atom types. Actually, the main target of MLMD are systems with three atom types. This is a serious problem for MD simulations of clay minerals, as they typically contain more than four atom types, e.g., silicon, aluminium, oxygen, hydrogen, and some cations.

In this presentation, we report our MLMD simulations of some clay minerals with four or five atom types. We succeeded in using MLMD to simulate all of them. We found that preparation of appropriate training data is crucial to make accurate PESs. We report evaluations of some physical quantities of the clay minerals.

\section{References}

[1] J. Behler and M. Parrinello, Phys. Rev. Lett. 98, 146401 (2007).

[2] A.P. Bartók et al., Phys. Rev. Lett. 104, 136403 (2010). 